\title{
Autocorrección para el aprendizaje y el fomento de la autonomía
}

\author{
SUSANA SANDUVETE-CHAVES \\ Universidad de Sevilla \\ Dpto. de Psicología Experimental \\ sussancha@us.es \\ ORCID: https://orcid.org/0000-0001-8568-6168 \\ D.O.I.: http://dx.doi.org/10.12795/JDU.2018.i01.46 \\ Pp.: 816-831
}

\section{Resumen}

El objetivo de este Ciclo de Mejora Docente fue ampliar el grado de implicación del alumnado con el fin de obtener un aprendizaje más profundo y duradero en el tiempo y aumentar su autonomía. La estrategia utilizada fue trabajo en grupo y autocorrección por parte del alumnado (tras un intercambio, para que nadie evaluara su propio trabajo). El alumnado participó en el Ciclo y adquirió en general los contenidos planificados, dándose diferencias significativas entre las respuestas más frecuentes en el pre-test (antes del curso, donde se daban más errores y omisiones) y en el post-test. Para cursos futuros, se esperan mantener las autoevaluaciones.

Palabras clave: Diseño y Análisis de Datos en Psicología I, Grado en Psicología, docencia universitaria, experimentación docente universitaria, autocorrección. 


\section{Breve descripción del contexto}

Realicé el Ciclo de Mejora Docente en una asignatura de primero del grado en Psicología denominada Diseño y Análisis de Datos en Psicología I. Se trataba de un grupo de mañana, donde las clases se impartieron en inglés. Del mismo modo, materiales y evaluaciones estuvieron en inglés. La mayor ventaja de este grupo con respecto a los demás es el reducido número de alumnos puesto que, por normativa, está limitado a 40 estudiantes, mientras que en los demás puede haber hasta el doble. Esto facilitó la coordinación de trabajos y la resolución de dudas.

Mi objetivo el año pasado fue, en esta misma asignatura, profundizar en la transferencia de lo aprendido a la práctica, puesto que las asignaturas como éstas, basadas en la estadística, pueden resultar abstractas y poco atrayentes para el alumnado. Se obtuvieron adecuados niveles en aprendizaje, aunque sólo un nivel de transferencia parcial (Sanduvete-Chaves, 2017). Una posible actuación para mejorar esta situación sería ampliar el grado de implicación del alumnado, y éste fue el objetivo para el presente trabajo.

\section{Diseño Previo del Ciclo de Mejora Docente}

\section{Modelos metodológicos}

A continuación, se presentan el modelo metodológico habitual, el ideal y el posible (de Alba y Porlán, 2017). El modelo metodológico habitual, seguido en cursos anteriores, consistía en que el alumnado realizaba las actividades propuestas por el profesorado, ya fuera en las clases prácticas o fuera del horario establecido para las clases. Una vez realizadas, el profesorado era el encargado de corregirlo. 
Mi modelo metodológico ideal supondría aumentar la implicación del alumnado en su propio aprendizaje; darles las riendas de la clase: que los propios estudiantes enseñaran a sus propios compañeros; que la misma clase propusiera actividades que les resultara interesantes para aplicar en el horario docente.

Respecto a mi modelo metodológico posible, ciertas preocupaciones me hicieron tender a ser moderada: había un temario que impartir necesariamente, dado que en segundo curso se continuaba con la siguiente parte de esta misma asignatura, Diseño y Análisis de Datos en Psicología II, por lo que las bases habían de consolidarse a lo largo del curso. Esto quería decir que las actividades no podían alargarse eternamente; que había que llevar un control de su duración para seguir avanzando según lo planificado. Una intervención intermedia sería aumentar gradualmente la implicación del alumnado. Y en el presente curso se persiguió pasar de una evaluación de los ejercicios por parte del profesorado a una autoevaluación. Al igual que cuando era el profesorado el que corregía los ejercicios, la autoevaluación sólo valía en la calificación un máximo de 0,5 sobre 10. Sin embargo, este medio punto era una pequeña recompensa (casi una excusa) para que el alumnado ejerciera un papel más activo. La hipótesis de partida fue que, de este modo, adquiririan los conocimientos de una manera más profunda, por lo que podrían extrapolar lo aprendido con mayor facilidad a diversas situaciones. Se controló la participación en la actividad con la asistencia (hojas de firma).

Este modelo metodológico posible constó de cuatro fases:

1. Se conformaron grupos de estudiantes de entre 3 a 5 personas. Estos grupos realizaron en conjunto el desarrollo y resolución de cada ejercicio.

2. Al finalizar cada ejercicio, se intercambiaron los desarrollos y resultados entre los grupos y ellos mismos se autoevaluaron. 
3. Seguidamente, el profesorado realizó una verificación del proceso y las autoevaluaciones realizadas por el alumnado.

4. Se realizó una puesta en común de todo proceso en cada ejercicio en dos fases: 4.1. En primer lugar, entre los grupos de estudiantes; y 4.2. Posteriormente, todo el grupo de clase con el profesorado.

En la Figura 1, se presenta esquemáticamente el modelo metodológico actual, el ideal y el modelo al que se aspiró con este ciclo de mejora.

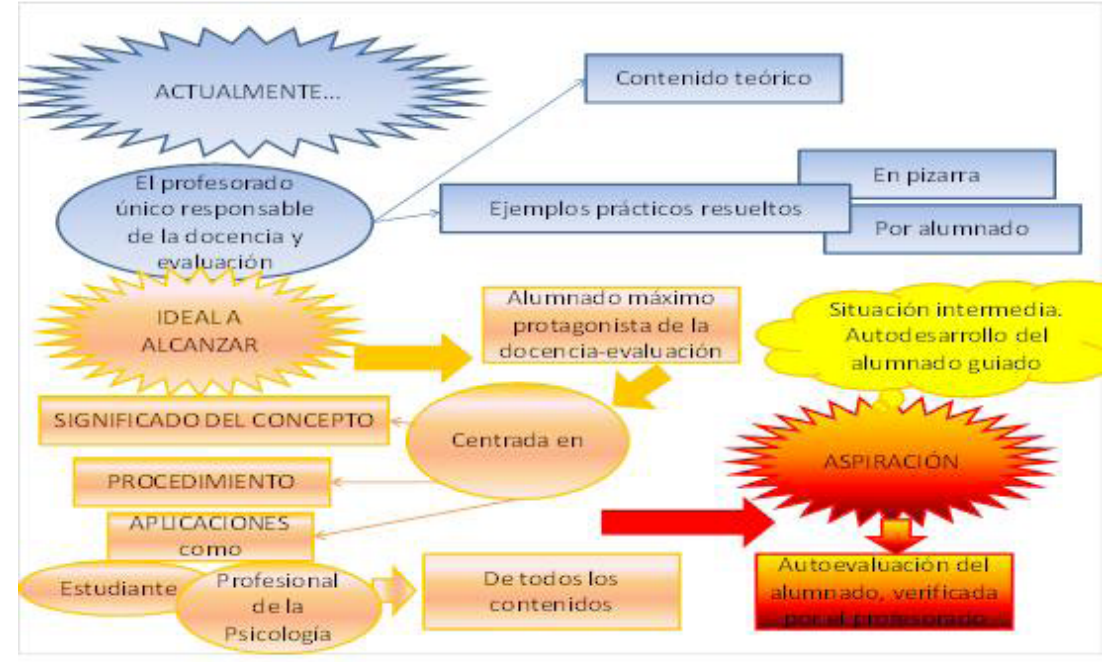

Figura 1. Modelo metodológico actual, modelo ideal y modelo realista al que se aspira.

\section{Mapa de contenidos y problemas}

De manera sintética en la Figura 2, se presenta el mapa de contenidos (García-Díaz, Porlán y Navarro, 2017) que el alumnado trabaja a lo largo del curso.

Jornadas de Formación e Innovación Docente del Profesorado I № 1 (2018) Reconocimiento-NoComercial-SinObraDerivada Internacional (CC BY-NC-ND 4.0.) 


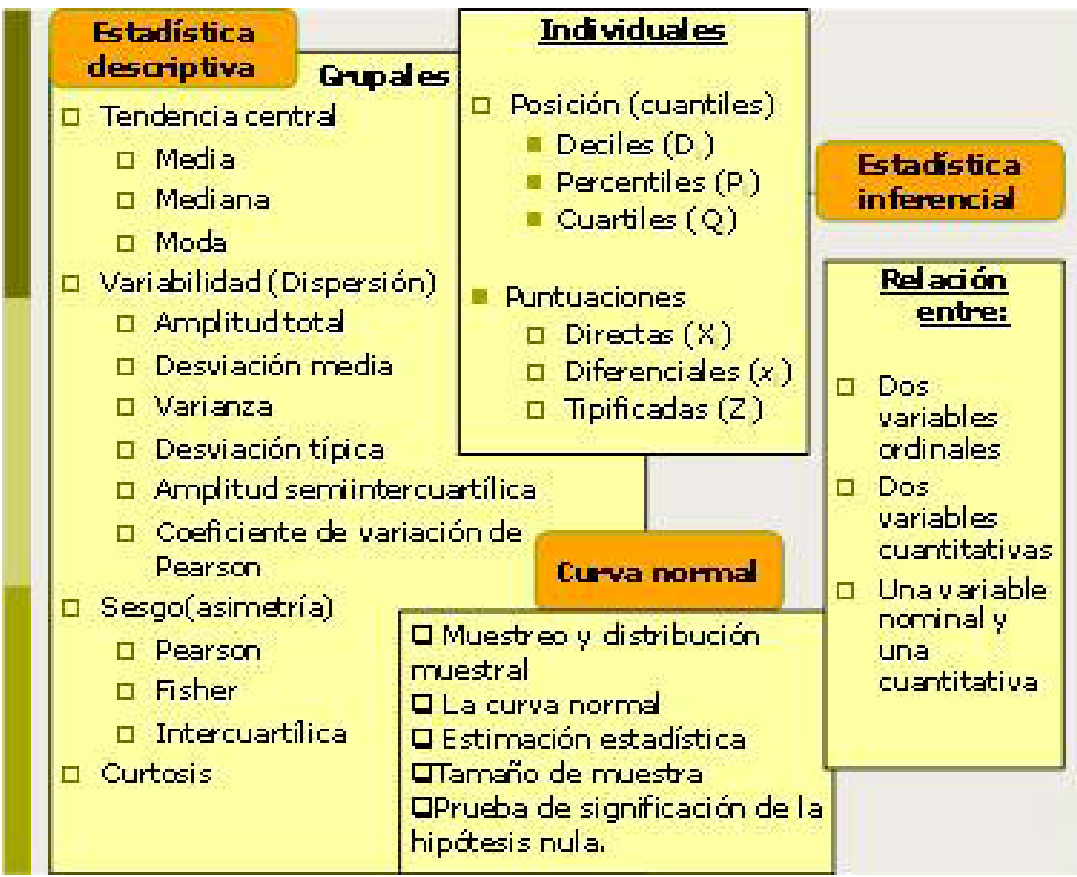

Figura 2. Mapa de contenidos a trabajar en la asignatura.

Las preguntas problema asociados a estos contenidos serían los siguientes:

1. ¿Cómo se calcula la amplitud semi-intercuartílica?

2. ¿Qué representa la moda?

3. ¿Cómo se interpreta la simetría?

4. ¿Cómo se calculan los percentiles?

5. ¿Cómo se interpretan los percentiles?

6. ¿Cómo se calcula el error máximo?

7. ¿Qué tipo de variables relacionan la chi cuadrado?

8. ¿Cuándo es estadísticamente significativo el resultado de la chi cuadrado?

9. Si un resultado es significativo y el tamaño de efecto es grande, ¿podemos decir que el efecto probablemente existe? Razona la respuesta.

La Figura 3 muestra la relación entre los contenidos a impartir y las preguntas problema propuestas: 


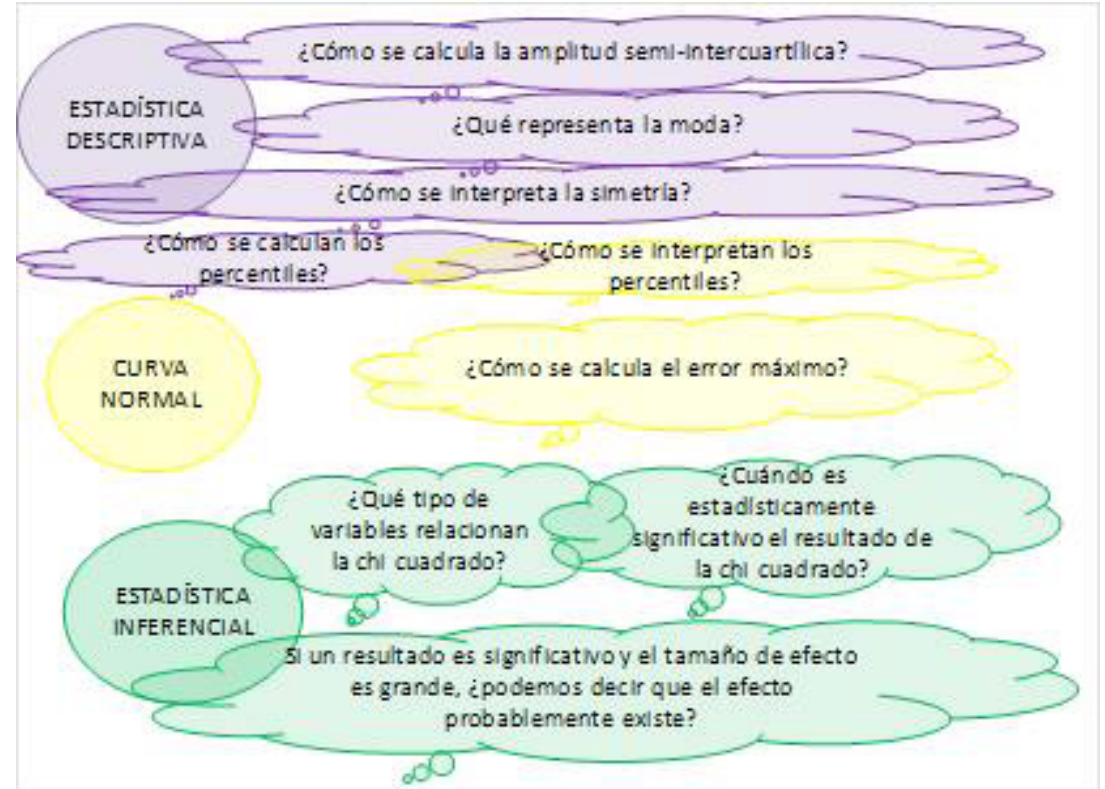

Figura 3. Relación entre los contenidos a impartir y las preguntas problema.

\section{Cuestionario inicial - final}

Al inicio del Ciclo de Mejora se presentaron las preguntas (ver Anexo). Las respuestas se clasificaron desde las más simples a las más completas y se calculó el porcentaje de alumnado que se ubicó en cada respuesta, creándose así la escalera de aprendizaje inicial (Rivero y Porlán, 2017). Al aplicar las mismas preguntas después del ciclo de mejora, se esperó que subieran en la escalera de conocimientos.

\section{Secuencia de actividades}

A partir de las ideas e hipótesis de partida de los estudiantes, así como de la conexión entre contenidos y preguntas problemas de la asignatura, planteamos una secuencia de actividades concretadas en ejercicios específicos para que el alumnado lograra adquirir los conocimientos previstos fomentando la autoevaluación. 
El modelo metodológico propuesto que constó de las cuatro fases generales previamente comentadas se repitió para cada uno de los siguientes ejercicios propuestos (se presentan en inglés, tal y como se trabajaron en clase):

\section{Exercise 1.}

\section{Statistics}

Anxiety

N

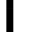
Mean

Mode

Std. Deviation

Skewness

Std. Error of Skewness

Kurtosis

Std. Error of Kurtosis

Percentiles

\section{Valid \\ Missing}

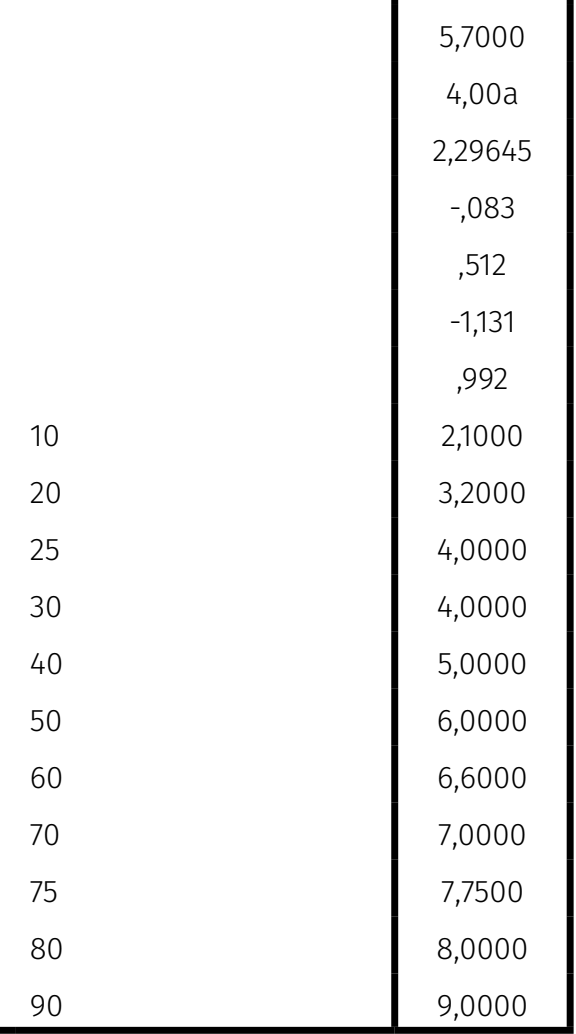

a. Multiple modes exist. The smallest value is shown

a) Calculate the semi-interquartile amplitude of anxiety. 
b) Which is the most frequent value?

c) Does anxiety present a symmetric distribution? Explain your answer.

Exercise 2. Scores obtained in an abstract reasoning test $(X)$ by a group of 100 students are normally distributed, with a standard deviation of 37.3. There are 25 students below score 65 and 25 students who over the 115 score.

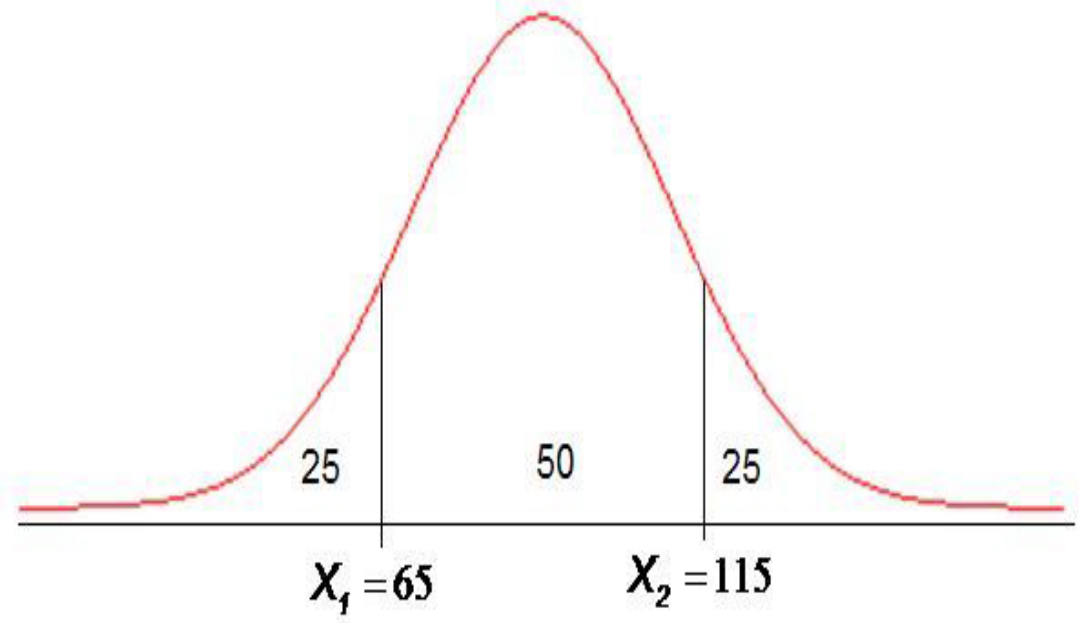

Calculate:

a) The mean in abstract reasoning.

b) $P_{79}$

c) How many persons obtained a score lower than 100 in the abstract reasoning test?

\section{Exercise 3.}

\section{Chi-Square Tests}

\begin{tabular}{|l|l|l|l|}
\hline & Value & Df & Asymp. Sig. (2-sided) \\
\hline Pearson Chi-Square & $3.069^{\mathrm{a}}$ & 2 & .216 \\
Likelihood Ratio & 3.136 & 2 & .208 \\
Linear-by-Linear Association & 2.468 & 1 & .116 \\
N of Valid Cases & 20 & & \\
\hline
\end{tabular}

a. 6 cells $(100,0 \%)$ have expected count less than 5 . The minimum expected count is 2,70 .

Jornadas de Formación e Innovación Docente del Profesorado | № 1 (2018) Esta obra se distribuye con la licencia Creative Commons 
gender * social_status Crosstabulation

Count

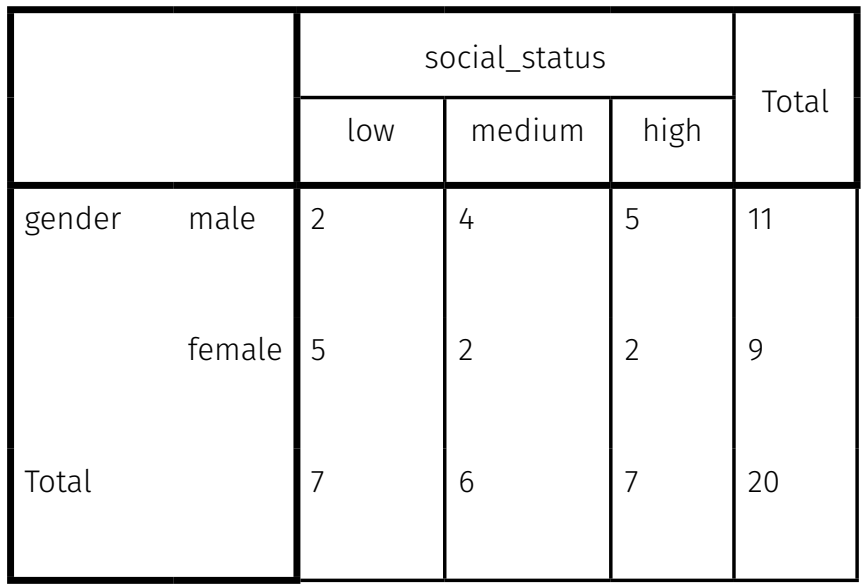

a) Which design do these statistical results come from? What statistical test has been used?

b) Interpret statistical results: Are there statistical differences? Interpret results in a substantive way.

Exercise 4. We obtained $a r_{x y}=0.67$ in 35 participants. Can we say that an effect probably exists? $(\alpha=0.05)$

Exercise 5. What is the maximum error to estimate the mean in a random sample of 100 participants, with a standard deviation of 4 and a confidence level of .95?

\section{Implementación del Ciclo de Mejora Docente}

\section{Relato de las sesiones}

En líneas generales, las sesiones se realizaron como se esperaba. El alumnado se conformaba en grupos. Algunas personas tendían a tomar siempre en el grupo a los mismos compañeros; otros iban cambiando cada día. Se

Jornadas de Formación e Innovación Docente del Profesorado | № 1 (2018) Esta obra se distribuye con la licencia Creative Commons 
planteaba un trabajo. Cada grupo trataba de resolverlo. Se les decía que, si no sabían cómo continuar, podían preguntar a otros grupos y, en última instancia, a la profesora. Una vez pasado el tiempo establecido (alrededor de $10 \mathrm{mi}-$ nutos, aunque dependía de la dificultad del ejercicio), la profesora recogía los ejercicios realizados, y los intercambiaba, de tal manera que un grupo no corregiría su propio trabajo. Un grupo voluntario lo corregía en público. Los demás aportaban posibles mejoras y preguntaban dudas, mientras que la profesora supervisaba que todo fuera correcto. Una vez corregidos todos los trabajos por parte del alumnado, la profesora volvía a intercambiar los ejercicios para que volvieran a quienes lo habían realizado. Cada grupo revisaba las correcciones que habían recibido. Si había algo que no veían claro, preguntaban a quienes les habían hecho las correcciones. La profesora revisaba en todo momento.

\section{Evaluación del aprendizaje del alumnado}

La Tabla 1 muestra la frecuencia de respuestas que cada alumnado dio para cada pregunta. Es interesante tener en cuenta que las respuestas aparecen ordenadas desde la más completa hasta la menos adecuada simbolizando la escalera de aprendizaje (Rivero y Porlán, 2017), de tal modo que se esperarían porcentajes más altos en las mejores respuestas tras el ciclo de mejora.

Jornadas de Formación e Innovación Docente del Profesorado | № 1 (2018) Esta obra se distribuye con la licencia Creative Commons 
Tabla 1. Comparación de respuestas dadas por el alumnado antes y después del Ciclo de Mejora

\begin{tabular}{|l|l|l|}
\hline Pregunta realizada & \%pretest & \%post-test \\
\hline 1. ¿Cómo se puede calcular la amplitud & & \\
semi-intercuartil? & 10 & 96,2 \\
a. Fórmula correcta & 3,3 & 0 \\
b. Usando cuartiles (pero no especifica cómo & 86,7 & 3,8 \\
exactamente) & & \\
c. No hay respuesta o fórmula incorrecta & & \\
\hline 2. ¿Qué es la moda? & & \\
a. El valor que más se da en una muestra & 96,7 & 100 \\
b. Un índice de tendencia central & 3,3 & 0 \\
\hline 3. ¿Cómo se puede interpretar el índice de & & \\
simetría? & 3,3 & 11,5 \\
a. S = 0 simétrica; S 0 negativa; S > positiva y & 3,3 & 30,8 \\
representación gráfica correcta & 3,3 & 6,7 \\
b. Misma interpretación (correcta), pero sin repre- & 0 & 34,6 \\
sentación gráfica & 0 & 3,8 \\
c. Interpreta correctamente positivo y negativo; no & 90,1 & 12,6 \\
menciona el 0 & & \\
d. S = 0 simétrica (o no se explica lo demás o está & & \\
incorrecto) & & \\
e. Indica el grado de simetría (sin especificar más) & & \\
f. No hay respuesta o respuesta incorrecta & & \\
\hline 4. ¿Cómo se puede calcular un percentil? & & \\
a. Fórmulas del percentil con y sin intervalo & 3,3 & 12,6 \\
b. Fórmula del percentil con intervalo & 0 & 12,6 \\
b. Fórmulas del percentil cuando no hay intervalo & 6,6 & 7,7 \\
c. Fórmula de la posición cuando no hay intervalo & 10 & 63,3 \\
o cuando hay & 80,1 & 3,8 \\
d. No hay respuesta o respuesta correcta (defini- & & \\
ción de decil) & 3,3 & \\
\hline 5. ¿Cómo se interpretaría un percentil? & & \\
a. Valor que deja bajo sí a un determinado por- & 16,7 & 46,1 \\
centaje de la muestra & 3,3 & 23,1 \\
b. División de la distribución en 100 partes & 3,3 & 23,1 \\
c. Posición de un valor & \\
d. Distribución dividida en partes & \\
e. No hay respuesta o respuesta incorrecta & \\
\hline
\end{tabular}

Jornadas de Formación e Innovación Docente del Profesorado | № 1 (2018) Esta obra se distribuye con la licencia Creative Commons Reconocimiento-NoComercial-SinObraDerivada Internacional (CC BY-NC-ND 4.0.) 


\begin{tabular}{|c|c|c|}
\hline $\begin{array}{l}\text { 6. ¿Cómo se calcularía el error máximo? } \\
\text { a. Fórmula correcta } \\
\text { b. Hay que utilizar el nivel de confianza } \\
\text { c. No hay respuesta o respuesta incorrecta (e.g., } \\
\text { standard error) }\end{array}$ & $\begin{array}{l}13,3 \\
3,3 \\
83,4\end{array}$ & $\begin{array}{l}88,5 \\
0 \\
11,5\end{array}$ \\
\hline $\begin{array}{l}\text { 7. ¿Qué tipo de variables se relacionan usando Chi } \\
\text { cuadrado? } \\
\text { a. Cualitativas y ordinales } \\
\text { b. Cualitativas } \\
\text { c. No hay respuesta o respuesta incorrecta } \\
\text { (cuantitativas) }\end{array}$ & $\begin{array}{l}0 \\
0 \\
100\end{array}$ & $\begin{array}{l}30,8 \\
46,1 \\
23,1\end{array}$ \\
\hline $\begin{array}{l}\text { 8. ¿Cuándo es estadísticamente significativo un re- } \\
\text { sultado obtenido usando Chi cuadrado? } \\
\text { a. Cuando es mayor que el valor de la tabla } \\
\text { b. Cuando es mayor que } 5 \text { (valor de la tabla para } \\
\text { un } 2 \times 2 \text { ) } \\
\text { c. Cuando se rechaza la hipótesis nula } \\
\text { d. No hay respuesta o respuesta incorrecta (e.g., al } \\
\text { revés) }\end{array}$ & $\begin{array}{l}0 \\
0 \\
0 \\
100\end{array}$ & $\begin{array}{l}30,8 \\
15,4 \\
7,7 \\
46,1\end{array}$ \\
\hline $\begin{array}{l}\text { 9. Si un resultado es significativo, y el tamaño del } \\
\text { efecto es grande, ¿podemos decir que probable- } \\
\text { mente existe el efecto? } \\
\text { a. Sí (correcto) y con razonamiento completo } \\
\text { b. Sí (correcto) pero con razonamiento incompleto } \\
\text { c. No hay respuesta o respuesta incorrecta }\end{array}$ & $\begin{array}{l}0 \\
3,3 \\
96,7\end{array}$ & $\begin{array}{l}76,9 \\
0 \\
23,1\end{array}$ \\
\hline
\end{tabular}

La participación en los cuestionarios fue elevada. De los 34 matriculados en el grupo, 30 personas cumplimentaron el pre-test, mientras que 26 cumplimentaron el posttest (fue al final de curso; cuando tienen cerca la fecha de los exámenes, tienden a ausentarse de las clases más a menudo en general). También fue elevada la asistencia a clase, de tal manera que 32 de los 34 matriculados asistieron al menos al 80\%. Por tanto, quienes respondieron a los cuestionarios, participaron en el Ciclo de Mejora.

En relación al aprendizaje del alumnado cabe destacar que, tal y como se aprecia en la Tabla 1, los porcentajes mejoraron en todos los casos. Se detectó una pregunta fácil, la 2 (sobre la moda), ya que casi todos los participantes respondieron correctamente en el pre-test. Para el resto de preguntas, el porcentaje más alto en el pretest se encontraba en el primer peldaño de la escalera, 
respondiendo que no lo sabía o dando una respuesta incorrecta. En el post-test, las preguntas 1, 2 y 6 obtuvieron una mayoría muy ventajosa en la alternativa más completa en comparación con las demás. En las demás preguntas, el alumnado estuvo más distribuido entre las diferentes respuestas, aunque siempre mejor que en el pre-test.

\section{Evaluación del Ciclo de Mejora Docente}

En líneas generales, puede afirmarse que el ciclo de Mejora se realizó como se esperaba, sin trabas importantes. Las mayores polémicas surgieron cuando un ejercicio podía resolverse con varios procedimientos o diferentes fórmulas, razón por las que los grupos evaluaban los ejercicios de sus compañeros como erróneos, cuando a veces estaban bien. Finalmente, todo quedaba aclarado.

Para el próximo curso, esperamos mantener la dinámica de la autocorrección puesto que para corregir, primero hay que aprender y comprender. Para evitar que otros posibles procedimientos fueran corregidos como incorrectos, se podría: (a) dar en clase todos los posibles procedimientos; y (b) plantear antes de la ejecución del ejercicio qué procedimiento seguir, para que todo el alumnado realice el mismo.

Los principios didácticos en los que nos hemos basado para la realización de este ciclo de mejora han sido los siguientes (García-Pérez y Porlán, 2017): (a) autonomía: a lo largo del grado, el alumnado ha de adquirir la autonomía suficiente como para resolver los problemas que les vayan surgiendo en el mundo laboral; y (b) aprendizaje significativo: atender a unas explicaciones de manera pasiva no es la mejor manera de adquirir conocimientos de manera duradera; hacer que el alumnado adquiera un rol activo en su propio aprendizaje y el de sus compañeros hará que éste sea más profunde y perdure más en el tiempo. 


\section{Referencias bibliográficas}

de Alba, N., y Porlán, R. (2017). La metodología de la enseñanza. En R. Porlán (Coord.), Enseñanza Universitaria. Cómo mejorarla (pp. 37-53). Madrid, España: Morata.

García-Díaz, E., Porlán, R. y Navarro, E. (2017). Los fines y los contenidos de enseñanza. En R. Porlán (Coord.), Enseñanza Universitaria. Cómo mejorarla (pp. 55-72). Madrid, España: Morata.

García-Pérez, F. y Porlán, R. (2017). Los principios didácticos y el modelo didáctico personal. En R. Porlán (Coord.), Enseñanza Universitaria. Cómo mejorarla (pp. 93-104). Madrid, España: Morata.

Rivero, A, y Porlán, R. (2017). La evaluación en la enseñanza universitaria. En R. Porlán (Coord.), Enseñanza Universitaria. Cómo mejorarla (pp. 73-91). Madrid, España: Morata.

Sanduvete-Chaves, S. (2017). Transferencia del conocimiento en análisis de datos. Comunicación presentada en las IV Jornadas de Formación e innovación docente. Sevilla, España: ICE

Jornadas de Formación e Innovación Docente del Profesorado | № 1 (2018) Esta obra se distribuye con la licencia Creative Commons 
Anexo. Cuestionario inicial-final aplicado

Name:

Please, answer the following questions.

\begin{tabular}{|l|l|}
\hline $\begin{array}{l}\text { How can you calculate the semi-interquartile } \\
\text { amplitude? }\end{array}$ & \\
\hline What is the mode? & \\
\hline How can you interpret de symmetry index? & \\
\hline How can you calculate a percentile? & \\
\hline How would you interpret a percentile? & \\
\hline How can you calculate the maximum error? & \\
\hline
\end{tabular}




\begin{tabular}{|l|l|}
\hline $\begin{array}{l}\text { Which kind of variables do you relate using } \\
\text { chi-squared? }\end{array}$ & \\
\hline $\begin{array}{l}\text { When is the result obtained in chi-squared sta- } \\
\text { tistically significant? }\end{array}$ & \\
\hline $\begin{array}{l}\text { If a result is significant and the effect size is big, } \\
\text { can we say that the effect probably exists? Exp- } \\
\text { lain your answer. }\end{array}$ & \\
\hline
\end{tabular}

